\title{
ZETA FUNCTIONS ON A CETAIN ORDERS IN A QUATERNION ALGEBRA
}

\author{
INSUK KIM $^{\mathrm{a}, *}$ AND SUNGTAE JUN ${ }^{\mathrm{b}}$
}

\begin{abstract}
There are several types of orders in a Quaternion algebra. Generally, zeta functions defined on orders of a Quaternion algebra give some informations on the ideal theory of orders. In this study, we investigate functional equalities between the zeta functions defined on orders of a Quaternion algebra.
\end{abstract}

\section{INTRODUCTION}

It is well known that the fundamental tool in the study of prime numbers is the Riemann zeta function. In a number field, Rieman zeta function is generalized and it is called Dedekind zeta function. This zeta function gives some informations on the ideal properties of the orders in the number field. More generally, if we consider the zeta functions on a quaternion algebra, then there are several ways to define zeta functions on it. In this paper, we will define a zeta function on a quaternion algebra as same manner as in [1] and we will study the properties of this zeta function. If $A$ is a quaternion algebra over $\mathbb{Q}$ and $\mathcal{O}$ is an order of $A$, then for a prime $p$ in $\mathbb{Q}$, $A \otimes \mathbb{Q}_{p}$ is either a division algebra or an algebra isomorphic to $2 \times 2$ matrix algebra over $\mathbb{Q}_{p}$. Then $\mathcal{O}$ is one of the following types: First, $A \otimes \mathbb{Q}_{p}$ is a division algebra and an order, $\mathcal{O} \otimes \mathbb{Z}_{p}$ in $A \otimes \mathbb{Q}_{p}$ is isomorphic to a subring which contains the ring of integers in a quadratic extension field of $\mathbb{Q}$. Second, $A \otimes \mathbb{Q}_{p}$ is an algebra isomorphic to $2 \times 2$ matrix algebra over $\mathbb{Q}_{p}$ and $\mathcal{O} \otimes \mathbb{Z}_{p}$ is isomorphic to a subring which contains $\mathbb{Z} \times \mathbb{Z}$. Finally, $A \otimes \mathbb{Q}_{p}$ is an algebra isomorphic to $2 \times 2$ matrix algebra over $\mathbb{Q}_{p}$ and $\mathcal{O} \otimes \mathbb{Z}_{p}$ is isomorphic to a subring which contains the ring of integers of a quadratic extension field of $\mathbb{Q}_{p}$. In [1], the first and the second cases were studied. Thus we will study the last case in this paper.

Received by the editors May 25, 2012. Revised August 11, 2012. Accepted August 13, 2012. 2000 Mathematics Subject Classification. 11R11, 11R52, 11R54.

Key words and phrases. zeta function, order, quaternion algebra.

* Corresponding author

The corresponding author's research was supported by Wonkwang University research grant, 2010. 


\section{Orders of Quaternion Algebra}

Let $A$ be a quaternion algebra over $\mathbb{Q}$, i.e. $A$ is a semi-simple algebra of dimension 4 over $\mathbb{Q}$. Then a lattice on $A$ is a finitely generated $\mathbb{Z}$ module containing a base of $A$ over $\mathbb{Q}$ and an order of $A$ is a lattice on $A$ which is also a subring with 1 . The analogous definitions hold for lattices and orders in $A_{p}=A \otimes \mathbb{Q}_{p}$ for a prime $p$. If $\mathcal{O}$ is an order of $A$, then $\mathcal{O}_{p}=\mathcal{O} \otimes \mathbb{Z}_{p}$ is an order of $A_{p}$.

It is well known that for a rational quaternion algebra $A, A \otimes \mathbb{Q}_{p}$ is either a division algebra or an algebra isomorphic to $2 \times 2$ matrix algebra over $\mathbb{Q}_{p}$. If $A \otimes \mathbb{Q}_{p}$ is a division algebra, $p$ is called ramified, otherwise $p$ is called split (see [3]).

Proposition 2.1. For given square free numbers $D$ and $H$ which are relatively prime each other, let $A$ be a quaternion algebra ramified at a prime $p \mid D$ and $\infty$. Then an order $\mathcal{O}$ of $A$ is either

(a) $\mathcal{O}_{p}$ is maximal if $p \mid D$,

(b) for all $p \mid H, \mathcal{O}_{p}$ is isomorphic to $\left\{\left(\begin{array}{cc}a & b \\ p^{n} c & d\end{array}\right) \mid a, b, c, d \in \mathbb{Z}_{p}\right\}$ for an integer $n$,

(c) for all other $p, \mathcal{O}_{p}$ is isomorphic to the full matrix ring $M\left(2, \mathbb{Z}_{p}\right)$,

or

(a) $\mathcal{O}_{p}$ is maximal if $p \mid D$,

(b) for all $p \mid H, \mathcal{O}_{p}$ is isomorphic to a subring which contains the ring of integers in a quadratic extension field of $\mathbb{Q}_{p}$,

(c) for all other $p, \mathcal{O}_{p}$ is isomorphic to the full matrix ring $M\left(2, \mathbb{Z}_{p}\right)$.

Proof. See [3], or [5].

In [1], Eichler considered an order, so called Eichler order when $n=1$ in the first case at Proposition 2.1. That is, the second condition (b) of an order $\mathcal{O}$ of $A$ becomes that $\mathcal{O}_{p}$ is isomorphic to $\left\{\left(\begin{array}{cc}a & b \\ p c & d\end{array}\right) \mid a, b, c, d \in \mathbb{Z}_{p}\right\}$ for all $p \mid H$.

On this Eichler order $\mathcal{O}$, the zeta function is defined by the sum

$$
\zeta(s)=\sum_{M \subset \mathcal{O}} n(M)^{-2 s},
$$

where $M$ is an integral left $\mathcal{O}$ - ideals (See [1]).

Locally, it can be expressed as

$$
\zeta(s)=\prod_{p} \zeta_{p}(s)
$$


where $\zeta_{p}(s)=\sum\left(M_{p}\right)^{-2 s}$ is summed over all integral $M_{p}$ ideals in $\mathcal{O}_{p}$ whose norm are powers of the prime $p$.

Remark. Since zeta function on orders of a quaternion algebra is closely related with Riemann zeta function, we need its definition,

$$
\zeta_{\mathbb{Q}}(s)=\sum n^{-s}=\prod\left(1-p^{-s}\right)^{-1},
$$

where the sum is over all integers $n>0$ and the product is over all primes $p$. The following theorem treats an order which is the generalization of Eichler order. For the computational convenience, we restrict ourselves $D=q$ and $H=p$ case only.

Theorem 2.2. Let $\mathcal{O}$ be an order of a quaternion algebra $A$ with the following properties.

(i) $\mathcal{O}_{q}$ is the maximal oreder of $A_{q}$,

(ii) there is a prime number $p$ such that $\mathcal{O}_{p}$ is isomorphic to

$$
\left\{\left(\begin{array}{cc}
a & b \\
p^{n} c & d
\end{array}\right) \mid a, b, c, d \in \mathbb{Z}_{p}\right\} \text {, where } n \text { is a positive integer, }
$$

(iii) for all other prime number $l, \mathcal{O}_{l}$ is isomorphic to the full matrix ring $M\left(2, \mathbb{Z}_{l}\right)$.

Then

$$
\zeta_{\mathcal{O}}(s)=\zeta_{\mathbb{Q}}(2 s) \zeta_{\mathbb{Q}}(2 s-1)\left(1-q^{1-2 s}\right)^{-1} \sum_{k=1}^{n-1}\left(1+p^{2(n+k) s}\right)^{-1} .
$$

Proof. Let $\mathcal{O}_{q}$ be the maximal order. Then there is a unique prime ideal of norm $q^{k}$ for a positive integer $k$. Hence

$$
\zeta_{\mathcal{O}_{q}}(s)=\frac{1}{\left(1-q^{-2 s}\right)}
$$

If $\mathcal{O}_{l}$ is isomorphic to the full matrix ring $M\left(2, \mathbb{Z}_{l}\right)$, by the elementary divisor theorem, every ideal $\mathcal{O}_{l}$ of norm $l^{n}$ is of the form, $\mathcal{O}_{l}\left(\begin{array}{cc}l^{a} & r \\ 0 & l^{b}\end{array}\right)$ with $a+b=i$ and $0 \leq r \leq l^{a}$. Thus

$$
\begin{aligned}
\zeta_{\mathcal{O}_{l}}(s) & =\sum_{i=0}^{\infty} \sum_{a+b=i} \frac{l^{a}}{l^{2 i s}}=\sum_{i=0}^{\infty} \frac{1-l^{i+1}}{1-l} \frac{1}{l^{2 i s}} \\
& =\frac{1}{1-l}\left(\frac{1}{1-l^{-2 s}}-\frac{l}{1-l^{1-2 s}}\right) \\
& =\left(\frac{1}{1-l^{-2 s}}\right)\left(\frac{1}{1-l^{1-2 s}}\right) .
\end{aligned}
$$


If $\mathcal{O}_{p}$ is isomorphic to $\left\{\left(\begin{array}{cc}a & b \\ p^{n} c & d\end{array}\right) \mid a, b, c, d \in \mathbb{Z}_{p}\right\}$, let $a=p^{t} a^{\prime}$ and $c=p^{t+m} c^{\prime}$. Then, since $\operatorname{gcd}\left(a^{\prime}, p^{m} c^{\prime}\right)=1$, there exist $\alpha, \beta \in \mathbb{Z}_{p}$ such that $\alpha a^{\prime}+\beta p^{m} c^{\prime}=1$. If $m \geq 0$, take $U=\left(\begin{array}{cc}\alpha & \beta \\ -p^{n} p^{m} c^{\prime} & a^{\prime}\end{array}\right) \in \mathcal{O}_{p}^{\times}$and by the elementary divisor theorem, we have

$$
U\left(\begin{array}{cc}
a & b \\
p^{n} c & d
\end{array}\right)=\left(\begin{array}{cc}
A & B \\
0 & D
\end{array}\right)
$$

Multiplying $V=\left(\begin{array}{ll}1 & r \\ 0 & 1\end{array}\right)$ on the right, we can reduce $B(\bmod A)$ and $D$. So we can get

$$
U\left(\begin{array}{cc}
a & b \\
p^{n} c & d
\end{array}\right) V=\left(\begin{array}{cc}
A & 0 \\
0 & D
\end{array}\right)
$$

unless $A$ and $D$ are both divisible by $p$ while $B$ is not.

If $m<0,\left(\begin{array}{cc}0 & p^{-m} \\ p^{n} & 0\end{array}\right)\left(\begin{array}{cc}a & b \\ p^{n} c & d\end{array}\right)$ becomes the previous case, $m \geq 0$. That is,

$$
\begin{aligned}
\left(\begin{array}{cc}
0 & p^{-m} \\
p^{n} & 0
\end{array}\right)\left(\begin{array}{cc}
a & b \\
p^{n} c & d
\end{array}\right) & =\left(\begin{array}{cc}
p^{n-m} c & p^{-m} d \\
p^{n} a & p^{n} b
\end{array}\right) \\
& =\left(\begin{array}{cc}
p^{n-m} p^{t+m} c^{\prime} & p^{-m} d \\
p^{n} p^{t} a^{\prime} & p^{n} b
\end{array}\right) \\
& =\left(\begin{array}{cc}
p^{n+t} c^{\prime} & p^{-m} d \\
p^{n+t} a^{\prime} & p^{n} b
\end{array}\right) .
\end{aligned}
$$

Thus every ideal $\mathcal{O}_{p}$ is of the form, $U\left(\begin{array}{cc}0 & p^{k} \\ p^{n} & 0\end{array}\right) \mathcal{O}_{p}\left(\begin{array}{cc}p^{a} & 0 \\ 0 & p^{b}\end{array}\right)\left(\begin{array}{cc}1 & r \\ 0 & 1\end{array}\right)$ where $k=$ $1, \cdots, n-1, a+b=i, 0 \leq r \leq p^{a}$. Thus

$$
\zeta_{\mathcal{O}_{p}}(s)=\left(\frac{1}{1-p^{-2 s}}\right)\left(\frac{1}{1-p^{1-2 s}}\right) \sum_{k=1}^{n-1}\left(1+p^{2(n+k) s}\right)^{-1} .
$$

Therefore, combining the above three cases altogether gives us

$$
\zeta_{\mathcal{O}}(s)=\zeta_{\mathbb{Q}}(2 s) \zeta_{\mathbb{Q}}(2 s-1)\left(1-q^{1-2 s}\right)^{-1} \sum_{k=1}^{n-1}\left(1+p^{2(n+k) s}\right)^{-1} .
$$

Next, from the above theorem, we have the relation between zeta functions defined on a certain order in a quaternion algebra. 
Theorem 2.3. Let the notations be as in Theorem 2.2. Then

$$
\begin{gathered}
\zeta_{\mathcal{O}}(s)\left(1-q^{1-2 s}\right) \sum_{k=1}^{n-1}\left(1+p^{2(n+k) s}\right) \\
=\pi^{-2+4 s}\left[\Gamma\left(\frac{1-2 s}{2}\right) \Gamma(1-s)\right] /\left[\Gamma(s) \Gamma\left(\frac{2 s-1}{2}\right)\right] \\
\cdot \zeta_{\mathcal{O}}(1-s)\left(1-q^{2 s-1}\right) \sum_{k=1}^{n-1}\left(1+p^{2(n+k)(1-s)}\right) .
\end{gathered}
$$

Proof. It is well known that the functional equation of $\zeta_{\mathbb{Q}}(s)$ is as follows;

$$
\zeta_{\mathbb{Q}}(s)=\pi^{-\frac{1}{2}+s}\left[\Gamma\left(\frac{1-s}{2}\right) / \Gamma\left(\frac{s}{2}\right)\right] \zeta_{\mathbb{Q}}(1-s) .
$$

Then we have

$$
\begin{gathered}
\zeta_{\mathbb{Q}}(2 s)=\pi^{-\frac{1}{2}+2 s}\left[\Gamma\left(\frac{1-2 s}{2}\right) / \Gamma(s)\right] \zeta_{\mathbb{Q}}(1-2 s), \\
\zeta_{\mathbb{Q}}(2 s-1)=\pi^{-\frac{3}{2}+2 s}\left[\Gamma(1-s) / \Gamma\left(\frac{2 s-1}{2}\right)\right] \zeta_{\mathbb{Q}}(2-2 s) .
\end{gathered}
$$

By (2.1),

$$
\zeta_{\mathcal{O}}(s)=\zeta_{\mathbb{Q}}(2 s) \zeta_{\mathbb{Q}}(2 s-1)\left(1-q^{1-2 s}\right)^{-1} \sum_{k=1}^{n-1}\left(1+p^{2(n+k) s}\right)^{-1}
$$

and

$$
\zeta_{\mathcal{O}}(1-s)=\zeta_{\mathbb{Q}}(2-2 s) \zeta_{\mathbb{Q}}(1-2 s)\left(1-q^{2 s-1}\right)^{-1} \sum_{k=1}^{n-1}\left(1+p^{2(n+k)(1-s)}\right)^{-1}
$$

imply

$$
\begin{aligned}
& \zeta_{\mathcal{O}}(s)\left(1-q^{1-2 s}\right) \sum_{k=1}^{n-1}\left(1+p^{2(n+k) s}\right) \\
& =\zeta_{\mathbb{Q}}(2 s) \zeta_{\mathbb{Q}}(2 s-1) \\
& =\pi^{-2+4 s}\left[\Gamma\left(\frac{1-2 s}{2}\right) / \Gamma(s)\right] \cdot\left[\Gamma(1-s) / \Gamma\left(\frac{2 s-1}{2}\right)\right] \zeta_{\mathbb{Q}}(2-2 s) \zeta_{\mathbb{Q}}(1-2 s) \\
& =\pi^{-2+4 s}\left[\Gamma\left(\frac{1-2 s}{2}\right) \Gamma(1-s)\right] /\left[\Gamma(s) \Gamma\left(\frac{2 s-1}{2}\right)\right] \\
& \cdot \zeta_{\mathcal{O}}(1-s)\left(1-q^{2 s-1}\right) \sum_{k=1}^{n-1}\left(1+p^{2(n+k)(1-s)}\right) \cdot
\end{aligned}
$$


Next, we treat the second case in Proposition 2.1, and for this syudy we restrict ourselves for a rational quaternion algebra $A$ ramified precisely at one finite prime $q$ and $\infty$.

Fix a prime $p(\neq q)$ and let $L$ be a quadratic extension field of $\mathbb{Q}_{p}, \mathrm{O}_{\mathrm{L}}$ the ring of integers in $L$ and $P_{L}$ its the prime ideal.

Then $\left\{\left(\begin{array}{cc}\alpha & \bar{\beta} \\ \beta & \bar{\alpha}\end{array}\right) \mid \alpha, \beta \in L\right\}=L+\xi L$ is a quaternion algebra over $\mathbb{Q}_{p}$, where $\xi=\left(\begin{array}{ll}0 & 1 \\ 1 & 0\end{array}\right)$. Obiously, we can see that $\xi \alpha=\bar{\alpha} \xi, \xi^{2}=1$ and $\bar{\xi}=-\xi$ (see Proposition 2.1 in [3]). Hence, we can define the norm of an element in $A$ as its determinant.

Theorem 2.4. Let $A$ be a quaternion algebra over $\mathbb{Q}$. For an odd prime $p \neq q$, if an order $\mathcal{O}_{p}$ of $A_{p}$ contains $\mathrm{O}_{\mathrm{L}}$, then $\mathcal{O}_{p}$ is one of the followings.

(i) If $p$ is a unramified prime in $L, \mathcal{O}_{p}(\nu)=\mathrm{O}_{\mathrm{L}}+\xi P_{L}^{\nu}$.

(ii) If $p$ is a ramified prime in $L, \mathcal{O}_{p}(\nu)=\mathrm{O}_{\mathrm{L}}+(1+\xi) P_{L}^{\nu-1}$ or $\overline{\mathcal{O}_{p}}(\nu)=$ $\mathrm{O}_{\mathrm{L}}+(1-\xi) P_{L}^{\nu-1}$.

Here, $\nu$ is a nonnegative integer.

Proof. See [3].

Remark. If $p$ is a ramified prime in $L$, then for $\nu \geq 1, \mathcal{O}_{p}(\nu)=\mathrm{O}_{\mathrm{L}}+(1+\xi) P_{L}^{\nu-1}=$ $\overline{\mathcal{O}_{p}(\nu)}=\mathrm{O}_{\mathrm{L}}+(1-\xi) P_{L}^{\nu-1}=\mathrm{O}_{\mathrm{L}}+\xi P_{L}^{\nu-1}$.

For computational convenience, we will consider the case that $p$ is ramified in $L(p)$ where $L(p)$ is a quadratic extension field of $\mathbb{Q}_{p}$.

Definition 1. Let $A$ be a rational quaternion algebra ramified precisely at one finite prime $q$ and $\infty$. An order $\mathcal{O}$ of $A$ has level $\widetilde{N}=(q ; L(p), \nu)$ with $\nu \geq 1$ if

(i) $\mathcal{O} \otimes \mathbb{Z}_{l}$ is the maximal order of $A \otimes \mathbb{Q}_{l}$ for a prime $l(\neq p)$,

(ii) $\mathcal{O} \otimes \mathbb{Z}_{p}=\mathrm{O}_{\mathrm{L}}+\xi P_{L}^{\nu}$.

Let $\mathcal{O}$ be an order of level $N^{\prime}=(q, L(p), \nu)$ in $A$. A left $\mathcal{O}$ ideal $I$ is a lattice on $A$ such that $I_{p}=\mathcal{O}_{p} a_{p}$ (for some $a_{p} \in A_{p}^{\times}$) for all $p<\infty$. Two left $\mathcal{O}$ ideals $I$ and $J$ are said to belong to the class if $I=J a$ for some $a \in A^{\times}$. Analogously, right $\mathcal{O}$ ideals can be defined. The class number of left ideals for any order $\mathcal{O}$ of level $N^{\prime}$ is the number of distinct classes of such ideals as usual sense.

The norm of an ideal, denoted by $N(I)$, is the positive rational number which generates the fractional ideal, $\{N(a) \mid a \in I\}$ of $\mathbb{Q}$. The conjugate of an ideal $I$, denoted by $\bar{I}$, is given by $\bar{I}=\{\bar{a} \mid a \in I\}$. The inverse of an ideal, denoted by $I^{-1}$, is given by $I^{-1}=\{a \in A \mid I a I \subset I\}$. 
Theorem 2.5. Let $\mathcal{O}$ be an order of a quaternion algebra $A$ with level $\widetilde{N}=(q ; L(p), \nu)$. Then

$$
\zeta_{\mathcal{O}}(s)=\zeta_{\mathbb{Q}}(2 s) \zeta_{\mathbb{Q}}(2 s-1)(p-1)\left(1-p^{1-2 s}\right)\left(1+p^{-2 n s}\right)\left(1-q^{1-2 s}\right) .
$$

Proof. As in Theorem 2.2, if $\mathcal{O}_{q}$ is the maximal order in $A_{q}$,

$$
\zeta_{\mathcal{O}_{q}}(s)=\frac{1}{\left(1-q^{-2 s}\right)} \text {. }
$$

Next, if $\mathcal{O}_{l}$ is isomorphic to the full matrix ring $M\left(2, \mathbb{Z}_{l}\right)$, by the proof of Theorem 2.2 ,

$$
\zeta_{\mathcal{O}_{l}}(s)=\left(\frac{1}{1-l^{-2 s}}\right)\left(\frac{1}{1-l^{1-2 s}}\right) .
$$

Finally, if $\mathcal{O}_{p}=\mathcal{O} \otimes \mathbb{Z}_{p}=\mathrm{O}_{\mathrm{L}}+\xi P_{L}^{\nu}$, then every ideal of $\mathcal{O}_{p}$ is of the form, $\left(\alpha+\xi \pi_{L}^{\nu}\right) \mathcal{O}_{p}$

To compute the norm of ideal, it is enough to consider $N\left(\alpha+\xi \pi_{L}^{\nu}\right)=N(\alpha)-p^{\nu}=$ $p^{n}$ where $\pi_{L} \mathrm{O}_{\mathrm{L}}=P_{L}$. If $\nu>n$, there are $\left|\mathrm{O}_{\mathrm{L}} / P_{L}\right|-1=p-1$ elements for $\alpha$. If $\nu \leq n$, in order to be $N\left(\alpha+\xi \pi_{L}^{\nu}\right) \in p^{n} \mathbb{Z}, \alpha=\pi_{L}^{\nu}+\beta \pi_{L}^{n-\nu}$ with $\beta \in\left(\mathrm{O}_{\mathrm{L}} / P_{L}\right)^{\times}$. Therefore, there are $\left|\left(\mathrm{O}_{\mathrm{L}} / P_{L}\right)^{\times}\right|=p-1$ elements for $\beta$. Now,

$$
\begin{aligned}
\zeta_{\mathcal{O}_{p}}(s) & =(p-1) \sum_{k=1}^{n-1} \frac{1}{p^{-2 k s}}+2(p-1) \sum_{k=n}^{\infty} \frac{1}{p^{-2 k s}} \\
& =(p-1) \frac{1}{\left(1-p^{-2 s}\right)}+(p-1) p^{-2 n s} \frac{1}{\left(1-p^{-2 s}\right)} \\
& =\frac{(p-1)\left(1+p^{-2 n s}\right)}{\left(1-p^{-2 s}\right)} .
\end{aligned}
$$

From the above three cases, we have

$$
\zeta_{\mathcal{O}}(s)=\zeta_{\mathbb{Q}}(2 s) \zeta_{\mathbb{Q}}(2 s-1)(p-1)\left(1-p^{1-2 s}\right)\left(1+p^{-2 n s}\right)\left(1-q^{1-2 s}\right) .
$$

Analogously as in Theorem 2.3, we are able to find the functional equation between zeta functions using Theorem 2.5.

Theorem 2.6. Let the notations be as in Theorem 2.5. Then we have the following equation.

$$
\begin{aligned}
\zeta_{\mathcal{O}}(s)= & \frac{\left(1-p^{1-2 s}\right)}{\left(1-p^{2 s-1}\right)} \frac{\left(1-q^{1-2 s}\right)}{\left(1-q^{2 s-1}\right)} \frac{\left(1+p^{-2 n s}\right)}{\left(1+p^{-2 n(1-s)}\right)} \cdot \pi^{-2+4 s} \\
& \cdot\left[\Gamma\left(\frac{1-2 s}{2}\right) \Gamma(1-s)\right] /\left[\Gamma(s) \Gamma\left(\frac{2 s-1}{2}\right)\right] \zeta_{\mathcal{O}}(1-s) .
\end{aligned}
$$


Proof. From the equation (2.4),

$$
\zeta_{\mathcal{O}}(1-s)=\zeta_{\mathbb{Q}}(2-2 s) \zeta_{\mathbb{Q}}(1-2 s)(p-1)\left(1-p^{2 s-1}\right)\left(1+p^{-2 n(1-s)}\right)\left(1-q^{2 s-1}\right) .
$$

Combining this equation with $(2.2),(2.3)$ and (2.4), we have

$$
\begin{aligned}
\zeta_{\mathcal{O}}(s)= & \frac{\left(1-p^{1-2 s}\right)}{\left(1-p^{2 s-1}\right)} \frac{\left(1-q^{1-2 s}\right)}{\left(1-q^{2 s-1}\right)} \frac{\left(1+p^{-2 n s}\right)}{\left(1+p^{-2 n(1-s)}\right)} \cdot \pi^{-2+4 s} \\
& \cdot\left[\Gamma\left(\frac{1-2 s}{2}\right) \Gamma(1-s)\right] /\left[\Gamma(s) \Gamma\left(\frac{2 s-1}{2}\right)\right] \zeta_{\mathcal{O}}(1-s) .
\end{aligned}
$$

\section{REFERENCES}

1. M. Eichler: The basis problem for modular forms and the traces of Hecke operators. Springer-Verlag, Lecture Notes in Math. 320 (1972), 75-151.

2. R. Godement: Les fonctions $\zeta$ des algebras simples: I, II. Seminaire Bourbaki, 1958/1959.

3. S. Jun \& I. Kim: On the Orders in a quaternion algebra over a dyadic local field. Honam Math. J. 31 (2009), no. 4, 611-632.

4. _ : The Mass formula of orders over a dyadic local field. Comm. Korean Math. Soc. 20 (2005), 231-238.

5. A. Pizer: An Algorithm for Computing modular forms on $\Gamma_{0}(N)$. J. Algebra 64 (1980), 340-390.

6. H. Shimizu: On the traces of Hecke operators. J. Fac. Sci. Univ. Tokyo 10 (1963), 1-19.

7. _ : On zeta functions of quaternion algebra. Ann. of Math. 81 (1965), 166-193.

8. T. Tamagawa: On $\zeta$ functions of a division algebra. Ann. of Math. 77 (1963), 387-405.

a Department of Mathematics Education, Wonkwang University, Iksan, Jeonbuk 540749 , KOREA

Email address: iki@wonkwang.ac.kr

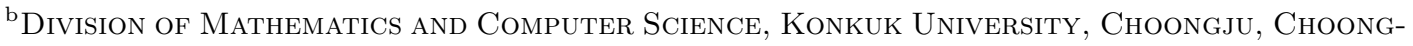
BUK 380-151, KOREA

Email address: sjun@kku.ac.kr 